\section{$\underset{\text { hommes }}{\text { \& migrations }}$}

\section{Hommes \& migrations}

Revue française de référence sur les dynamiques

migratoires

1285 | 2010

L'appel du pied

Les champions des cités

\title{
Les champions des cités
}

Parcours migratoires et effets de quartier

\section{William Gasparini}

\section{(2) OpenEdition \\ Journals}

\section{Édition électronique}

URL : http://journals.openedition.org/hommesmigrations/1186

DOI : 10.4000/hommesmigrations. 1186

ISSN : 2262-3353

Éditeur

Musée national de l'histoire de l'immigration

Édition imprimée

Date de publication : 1 mai 2010

Pagination : 108-123

ISSN : 1142-852X

Référence électronique

William Gasparini, «Les champions des cités », Hommes \& migrations [En ligne], 1285 | 2010, mis en

ligne le 29 mai 2013, consulté le 21 avril 2019. URL : http://journals.openedition.org/

hommesmigrations/1186; DOI : 10.4000/hommesmigrations.1186 


\section{Les champions des cités Parcours migratoires et effets de quartier}

Par William Gasparini, sociologue, professeur des universités en STAPS, directeur de l'équipe de recherche en sciences sociales du sport (EA 1342), Université de Strasbourg

En France, chaque grande vague d'immigration a donné son contingent de footballeurs d'exception. Des cités ouvrières aux banlieues des grandes

métropoles, la carte de leur répartition géographique reproduit

assez fidèlement celle de l'implantation des communautés immigrées sur le territoire français. Tous issus de quartiers populaires, les footballeurs

d'origine étrangère n'ont pourtant pas vécu les mêmes expériences sportives, urbaines, sociales, culturelles et immigrées. De la Lorraine à Marseille en passant par Vénissieux, trajectoires croisées de trois artistes du ballon rond. 
Bien que portant le même maillot bleu, Michel Platini ${ }^{(1)}$ Luis Fernandez $^{(2)}$ ou Zinédine Zidane ${ }^{(3)}$ appartiennent d'abord à des âges différents du football et de l'immigration. Platini, de la troisième génération d'une famille immigrée italienne (son grand-père s'est fixé à Jceuf, en Lorraine, dans les années vingt), représente le dernier fleuron du football des corons miniers. Né à Tarifa (Espagne), Luis Fernandez est, quant à lui, issu de la vague d'immigration qui arrive en France dans les années soixante. Élevé dans la ZUP des Minguettes à Vénissieux, il est le pur produit des nouvelles banlieues populaires. Fernandez obtient la naturalisation française en 1981 pour pouvoir jouer en équipe de France. Né à Marseille dans le quartier multiculturel et ouvrier de la Castellane, Zinédine Zidane appartient enfin à la deuxième génération issue de l'immigration algérienne postcoloniale.

À travers l'évocation de ces trois champions, on peut voir l'exceptionnelle contribution de l'immigration au football français. Pourtant, parmi eux, seul Fernandez n'est pas né sur le sol français. Michel Platini rappelle dans un entretien qu'il s'est toujours senti français : "Un jour, j’étais reçu par un adjoint au maire à Belfort en tant qu'entraîneur de l'équipe de France. Dans son discours, l'élu a parlé de moi comme d'un bon exemple d'intégration. J'ai été très surpris parce que je ne me suis jamais considéré comme étranger. Je n'avais jamais parlé italien, mon père non plus. Mon grand-père parlait lui aussi français. Je suis de la troisième génération ${ }^{(4)}$ " De même, les origines kabyles du Français Zidane et son attachement à la "mère patrie" algérienne ne sont rappelés que tardivement, à l'occasion du match France-Algérie en 2001. Les médias le sollicitent alors fortement sur ses origines et ses sentiments "patriotiques" et, à force d'être interrogé par les journalistes sur ses racines algériennes, il concède qu'il aura un "pincement au cœur" en rentrant sur le terrain.

On le voit, l'absence de définition précise du terme "immigré" et l'instrumentalisation (même bienveillante) des origines risque non seulement de masquer les contrastes essentiels qui marquent les trajectoires des joueurs ainsi désignés, mais nous conduit aussi à oublier le poids des conditions sociales d'existence dans la production du talent sportif. Au-delà de leurs différentes origines nationales et de l'incorporation du parcours migratoire de leur famille, ces footballeurs ont un point commun : l'apprentissage du football dans les rues d'une cité populaire avant leur prise en charge par le club et l'institution sportive fédérale.

Pour comprendre les raisons qui expliquent l'influence conjointe de l'immigration et de la vie dans les quartiers populaires sur le football français depuis plus de soixantedix ans, il faut d'abord sortir des analyses superficielles sur "l'immigré qui réussit par la volonté de s'en sortir" pour étudier les trois âges de l'encadrement populaire par le football. Il est ensuite nécessaire de rappeler que l'espace des sportifs issus de l'immigration est lui-même situé dans l'espace social tout en étant marqué par l'empreinte des politiques publiques dites "d'intégration"(5)". 


\section{Occuper les ouvriers et les jeunes des cités industrielles}

En France, les immigrés représentent depuis longtemps une composante importante de la classe ouvrière, et plus particulièrement de l'univers des ouvriers les moins qualifiés. C'est pendant l'entre-deux-guerres que le football commence à se répandre dans les milieux ouvriers suite, notamment, à l'action décisive des milieux patronaux "paternalistes" qui voient dans ce sport collectif un moyen d'encadrement et d'"éducation" des salariés, mieux adapté aux nécessités de la grande usine que des activités plus individualistes, comme la gymnastique ${ }^{(6)}$.

Les enfants de l'immigration se retrouvent souvent dans les clubs sportifs, dont le développement est encouragé par les industriels, qui se lancent alors dans une campagne d'équipement en construisant des terrains de football et en aménageant des vestiaires. Ainsi en est-il du club de Jceuf dans lequel évolue le père de Michel Platini, situé dans le bassin houiller de Briey et dont les mines et l'usine sidérurgique appartiennent à l'une des grandes familles de l'acier, les Wendel. La diffusion du football parmi les ouvriers immigrés et leurs fils apparaît comme un bon moyen de réduire les tensions sociales. Instrument de discipline, de moralisation et de socialisation, le football permet aussi d'intégrer les immigrés plus étroitement à l'entreprise autour de laquelle se développent la solidarité et 1'“esprit maison"(7).

Le club de football joue enfin un rôle d'assimilation pour des ouvriers déracinés : la sociabilité de quartier se développe à travers les réseaux sportifs 
constitués dans les sociétés cyclistes ou de quille et les clubs de football. La cité ouvrière structure la vie économique, sociale et culturelle au travers de relations de voisinage, fondées sur l'homogénéité d'une "famille" élargie, premier élément d'une "conscience de classe" qui permet néanmoins de renouer pour des déracinés avec la sociabilité villageoise. École de moralité - au sens de Durkheim ${ }^{(8)}-$, le club de football fabrique l'attachement au groupe, l'ethos masculin et le sens de la compétition, dans une société très hiérarchisée sur le plan social. Pour les fractions populaires, l'appartenance au club devient aussi la marque tangible de l'acceptation dans une confrérie qui permet de s'arracher à l'anonymat de la masse et, partant, de s'attirer (si le niveau sportif est bon) l'admiration et l'assentiment de la société autochtone locale. Ainsi, par les rencontres qu'il suscite dans le cadre de la compétition, le football des cités industrielles conduit fréquemment à une interpénétration tant des communautés immigrées entre elles que des immigrés et des Français "de souche".

\section{Des cités ouvrières aux banlieues populaires}

Après la Seconde Guerre mondiale, que ce soit dans les mines de fer, dans le bassin houiller, dans l'industrie textile puis automobile, le football devient un loisir qui va faire partie intégrante de la vie culturelle des ouvriers issus de l'immigration. Les clubs des cités minières de Lorraine et du Nord vont ainsi fournir au football professionnel français de très nombreux joueurs d'origine italienne ou polonaise. $\mathrm{A}$ titre d'exemple, dans la même rue de la cité de Calonne sont nés Wisnieski, Budzinski, Synakowski, qui comptèrent 57 sélections en équipe de France à eux trois alors que la mine d'Auchel, dans le Pas-de-Calais, a donné une pléiade de grands joueurs professionnels ${ }^{(9)}$. Le magazine France football écrivait à ce sujet en 1955 : "L'élite du football français grandit à l'ombre des terrils"(10).

Avec les nouvelles vagues d'immigrants qui ont gagné la France à partir des années soixante (en provenance d'Algérie, mais aussi d'Espagne et d'Afrique noire), le développement économique de la France puis la crise des années soixante-dix, la carte du football ouvrier et immigré se déplace. Les nouveaux immigrants et leurs familles se sont dirigés vers les nouveaux lieux de concentration du prolétariat démuni : les grands centres urbains, c'est-à-dire le monde des ZUP et des grandes banlieues. Pour faire face à la crise du logement qui accompagne les trente glorieuses, les premières "tours" des grands ensembles apparaissent à proximité des villes de Paris, Lyon, Marseille. Avec l'anonymat propre aux grandes villes, le club 
de football n'est plus au centre de la sociabilité locale. Cependant, la nouvelle source de richesse du football français va désormais se situer dans les banlieues. Bien qu'ils n'appartiennent pas à la même génération de l'immigration, les exemples de Fernandez et de Zidane sont significatifs de ce changement.

\section{Endosser les stigmates du quartier}

Après la mort de son père (camionneur de profession) et un passage à Barcelone, Luis Fernandez s'installe en 1968 avec sa famille de six enfants au onzième étage d'une des premières tours édifiées dans le quartier des Minguettes. Symbole de la modernité, cette zone à urbaniser en priorité (ZUP) va pourtant progressivement devenir le symbole de l'exclusion urbaine. Dans son autobiographie, Luis Fernandez rappelle cette stigmatisation liée à la banlieue: "Dans toute la région, notre équipe avait mauvaise réputation. Partout on disait que les Minguettes, c'était le quartier des loubards. Les autres équipes avaient peur de venir chez nous et lorsque nous allions chez l'adversaire, nous étions accueillis presque comme des pestiférés ${ }^{(11)}$." Dans ce qu'il définit lui-même comme une "tour de Babel" qui reçoit, aux côtés des Espagnols et des Portugais, le flux migratoire croissant de Nord-Africains, Luis Fernandez apprend difficilement le français : l'espagnol est la langue familiale et le jeune Luis se rend en Espagne chaque été pendant les vacances ${ }^{(12)}$. Dans ces conditions, au collège, comme nombre d'enfants de migrants, il entre en sixième de transition avant d'être orienté rapidement vers une filière technique.

Quelques années plus tôt, au cours de l'hiver 1953, arrivait en France le père de Zidane, dans des conditions un peu différentes. Originaire du village d'Aguemoune en Kabylie (Algérie), Smaïl Zidane travaillait dans la culture et la vente des fruits et légumes produits sur le terrain familial ${ }^{(13)}$. À l'âge de dix-sept ans, il émigre vers la métropole pour fuir la misère dans ce qui était encore l'Algérie française. Pour de nombreux Algériens, "la seule porte qui reste, c'est la France... On dit qu'elle est le pays $d u$ bonheur ${ }^{(14)}$ !" Faisant partie de la première génération d'immigrés maghrébins venus travailler par nécessité, Smaïl Zidane arrive à Paris pour travailler comme manceuvre sur des chantiers de construction. Il mène alors la vie d'un travailleur immigré ordinaire, partageant un garni avec trois amis du village natal, faisant les trois-huit et expédiant la moitié de son salaire à ses parents ${ }^{(15)}$. En 1962, à l'heure de l'indépendance, il décide de rentrer au pays. Mais, avant de prendre le bateau, il séjourne pendant quelques semaines auprès de membres de sa famille à Marseille et rencontre sa future épouse, Malika, arrivée en France à l'âge de six ans, grâce à la politique de regroupement familial. Le couple Zidane s'installe à Marseille, dans la 
cité Brassens d'abord, puis à la Castellane (quartiers nord), où Yazid "Zinédine" a vu le jour en 1972. Après avoir joué au foot au pied des immeubles de la Castellane dès son plus jeune âge, Zinédine rejoint à dix ans un club de quartier, puis prend sa première licence à l'US Saint-Henri (le club où jouait son père) dans le nouveau contexte des années quatre-vingt. Avec la politique de développement social des quartiers (DSQ), puis la politique de la Ville, le "quartier" devient, sous l'appellation "quartier sensible", "quartier en difficulté" ou encore "quartier difficile", une nouvelle catégorie de l'action publique. C'est à partir de cette période que le sport devient l'un des moyens politiques d'encadrer la jeunesse (masculine) des banlieues.

\section{Encadrer les jeunes des quartiers par le sport}

La montée du chômage, l'arrivée de populations immigrées précarisées, le repli sur le quartier, l'émergence de nouvelles exclusions et la montée des violences urbaines à partir des années quatre-vingt conduisent les pouvoirs publics à utiliser le sport 
comme outil de développement social dans les banlieues ${ }^{(16)}$. Profitant de la médiatisation des figures immigrées de la réussite sportive, l'État puis les collectivités locales organisent, soutiennent financièrement les clubs et favorisent les pratiques sportives à caractère populaire à proximité des grands ensembles urbains. Sous l'impulsion conjointe des politiques

Sans cesse rappelée par les médias, l'origine territoriale - la cité - devient le symbole d'une probable réussite sportive, surtout dans des sports populaires comme la boxe ou le football. de la ville et du ministère de la Jeunesse et des Sports, de nouveaux dispositifs de prévention et d'encadrement de la jeunesse voient ainsi le jour, parmi lesquels les dispositifs "sociosportifs" à destination des "jeunes des cités" $"(17)$. Avec les sports de combat, le football est particulièrement intéressant pour les pouvoirs publics, car il attire nombre de garçons issus de l'immigration, permettant ainsi la mixité sociale prônée par le modèle républicain d'intégration, qui ne fonctionne souvent que dans les clubs sportifs et les collèges. Cependant, tout comme dans les banlieues, le brassage dans les clubs ne se réalise qu'entre populations des couches populaires habitant les cités.

Les politiques d'insertion par le sport peuvent être analysées comme une nouvelle forme d'encadrement et de contrôle des jeunes issus des classes populaires face aux effets non maîtrisés des politiques néolibérales amorcées en France au début des années quatre-vingt ${ }^{(18)}$. À partir de cette période, l'État est confronté à la fois à un chômage structurel élevé désorganisant les milieux populaires ouvriers et transformant les cités HLM en quartiers de relégation (sociale et urbaine), mais également aux effets du regroupement familial lié aux politiques d'immigration et faisant apparaître une jeunesse française de plus en plus "métissée" d'origines ethniques diverses. Mais les quartiers de grands ensembles ne sauraient simplement se caractériser par l'absence de sociabilité et d'identité collective. À travers les "souscultures urbaines" (qui vont du rap au basket de rue, en passant par le football de pied d'immeuble ou des clubs de quartier), mais aussi les regroupements à base ethnique, de nouvelles formes de solidarité et de pratiques culturelles se développent, entre liens communautaires et modes d'intégration de proximité. Après la victoire de l'équipe de France "Black-Blanc-Beur" au Mondial de football en 1998, le conseiller du ministre de l'Intérieur de l'époque déclarait : "Zidane a fait plus par ses dribbles et ses déhanchements que dix ou quinze ans de politique d'intégration ${ }^{(19)}$." L'immigré - et, plus généralement, le "jeune des quartiers qui a réussi grâce au sport" - apparaît ainsi comme la nouvelle figure médiatique qui émerge progressivement à partir de la fin des années quatre-vingt. Entre un Platini 
d'origine italienne et un Zidane des quartiers nord de Marseille, on peut observer un changement de regard de l'opinion : sans cesse rappelée par les médias, l'origine territoriale - la cité - devient le symbole d'une probable réussite sportive, surtout dans des sports populaires comme la boxe ou le football.

Les footballeurs "issus de l'immigration" et des banlieues, placés au centre de la construction médiatique de la figure de l'immigré, deviennent progressivement représentatifs des jeunes exclus du système éducatif mais "intégrés" par le sport. Pour certains, dotés d'aptitudes physiques et d'expériences corporelles accumulées dans les quartiers populaires, c'est dans le football qu'ils convertissent ce capital spécifique et qu'ils trouvent une promotion sociale de substitution. Moins riches en ressources économiques et culturelles, possédant de surcroît un capital symbolique "négatif" lié à la stigmatisation, ils trouvent dans le sport de haut niveau un espace qui reconnaît leur compétence et qui tire profit de dispositions, qualités et savoirs pratiques valorisés dans les milieux populaires.

\section{L'acquisition du capital sportif de rue}

On a souvent célébré chez les trois joueurs le "flair", 1'"intuition" et 1"“intelligence du jeu", qui seraient à la croisée d'une "nature" et d'une "culture de la rue". Il s'agit là d'une sorte d'idéologie du don qui est mise en avant au détriment de l'effort et du travail sportif pour acquérir ces compétences. Le niveau sportif acquis est en réalité la production d'une triple conjonction de facteurs : un rapport au monde transmis par la famille, une technique instituée, transmise par l'institution sportive (le club, le centre de formation, les stages de sélection...) et un rapport pratique au football acquis grâce à l'expérience du foot de rue et de pied d'immeuble dès le plus jeune âge. Considérer le "talent" comme un capital au sens de Bourdieu ${ }^{(20)}$ revient ainsi à l'analyser comme une compétence qui se transmet par le biais d'un héritage (familial ou de "grands frères"), qui s'active au travers d'expériences accumulées du quartier au club, et qui permet de dégager des profits selon l'opportunité qu'a son détenteur d'opérer les placements les plus rentables (accéder à un centre de formation puis à un club professionnel, par exemple).

Les trois footballeurs apprennent d'abord la technique du dribble dans la rue avant de se licencier en club. Fils d'Aldo Platini (joueur de football professionnel du FC Nancy puis entraîneur), Michel apprend d'abord le football dans la cité ouvrière de Jcuf, avec les quelques conseils du père. C'est sur la place Tartane, entourée des immeubles du quartier de la Castellane que le jeune Yazid jouait au 
football avec ses frères et copains du quartier après l'école et pendant les vacances. Dans les banlieues et les cités populaires, le football se structure souvent autour du duel entre des partenaires-adversaires le temps du jeu ${ }^{(21)}$. Parce qu'elle est propice à la réalisation de "petits ponts", de "roulades", de "râteaux" ou de "passements de jambe", la situation de "un contre un" rend possible la performance de rue et l'exploit. Mais le duel et l'affrontement peuvent aussi constituer un mode de rapport social privilégié chez des garçons et jeunes adultes exclus du système scolaire ou du monde du travail mais "intégrés" dans leur quartier. Depuis les années quatre-vingt, la vie quotidienne des "jeunes des cités" dans les banlieues se caractérise par l'intensification des rivalités pour le contrôle des ressources locales qui se sont raréfiées et le développement de microsociétés emboîtées (équipes, bandes et classes d'âge, principalement). D'après Thomas Sauvadet ${ }^{(22)}$, cette situation permet l'accumulation d'un capital spécifique aux banlieues que l'auteur a baptisé "capital guerrier". Ne se réduisant pas aux seules aptitudes mobilisables dans des situations violentes, ce capital est également formé par des ressources relationnelles, telles que la création et l'entretien d'un réseau ou la maîtrise de la "tchatche". Convertie dans le combat sportif (le match de football ou de basket), cette ressource permet aux jeunes d'être "agressifs" et respectés dans le jeu tout en respectant le code sportif.

\section{L'honneur chevillé au corps}

Dans ces quartiers de relégation, le corps est aussi le vecteur par lequel se construit une identité figurée. Pascal Duret a montré que si l'engagement corporel des classes populaires se dévalue à mesure que crôt le niveau d'instruction et de diplôme, des "espaces de jeu" célébrant la force subsistent cependant, notamment dans les cités ${ }^{(23)}$. Dans les banlieues, nombre de jeunes adolescents valorisent le corps musclé et sportif car il constitue un "capital". Les valeurs de virilité sont au principe de l'estime de soi et de la reconnaissance des autres. Quand Luis Fernandez dit que "le foot, ce fut la chance de ma vie ${ }^{(24)}$, il évoque autant un aiguilleur détournant des voies de la délinquance et des combats de rue qu'un formidable ascenseur social (avant d'être joueur professionnel, il occupait un emploi d'aide-électricien dans une chaudronnerie). La force physique en milieu populaire est d'autant plus sacralisée qu'elle constitue souvent (pour les garçons) l'ultime ressource qui puisse être mise en avant pour se définir socialement ${ }^{(25)}$. Le corps pour les ouvriers représente souvent la première des richesses et le premier réservoir de forces: "Il est outil de travail et principe d'affirmation de soi ${ }^{(26)}$." Pour les hommes, la rapidité des bagarres et la fréquence des coups découlent d'un état où la démonstration de force est encore 
la meilleure arme pour l'emporter dans un conflit. L'exemple du "coup de boule" de Zidane donné à l'Italien Materazzi en finale de la Coupe du monde de 2006 est assez significatif : rappelant le "code de l'honneur", ce geste fait immédiatement penser à certains "jeunes des quartiers" qui, lorsqu'on leur manque de "respect", n’hésitent pas à recourir à la force. Pour sauver son honneur, il faut se faire justice. Qu'il le veuille ou non,Zidane a fait passer un message : un homme insulté n'attend pas. Il faut frapper sinon l'absence de réaction justifie, dans cette logique, la domination ${ }^{(27)}$. Cet ethos de l'honneur dépasse les murs des stades et renvoie à des valeurs inculquées durant la socialisation. Avec le "coup de boule", l'icône des stades est redevenue un transfuge de classe issu de l'immigration et rattrapé par sa condition initiale.

François Dubet avait déjà remarqué la récurrence du thème de la "force" dans les discours des jeunes "galériens" de banlieues ${ }^{(28)}$. Seulement, il analysait la force comme une composante de la "rage", c'està-dire essentiellement comme un signe d'anomie et de désorganisation sociale et non pas comme un trait culturel spécifique. Cet attrait pour la puissance physique se retrouve aussi bien dans le style des consommations culturelles adolescentes (goût prononcé pour les personnages et héros à la force extraordinaire dans les jeux vidéo, à la télévision, dans les bandes dessinées...) que dans l'orientation du choix des pratiques sportives (musculation, sports de combat, sports collectifs "virils"). Christian Pociello l'avait déjà montré en 1981 : la force est l'une des quatre grandes catégories autour desquelles se structure l'espace des choix sportifs $^{(29)}$. 


\section{Du foot de pied d'immeuble au foot institué}

Parce que les "jeunes des cités" ne s'engagent que dans des pratiques où ils se reconnaissent a priori quelques compétences, cette proximité des pratiques, des ressources, des dispositions, des habitus requis permet de comprendre que les sports de rue en général, le football de pied d'immeuble en particulier, puissent être un moyen de "repêchage" et le vecteur d'une réhabilitation symbolique. Ensuite, de la rue au club, il n'y a qu'un pas à faire, surtout quand les copains le fréquentent aussi. Lintégration en club ne signifie pas pour autant l'abandon de la pratique autonome, qui ressource les joueurs dans un cadre plus convivial, moins sérieux. L'affiliation peut donc être double, à la fois dans le système fédéral et dans des groupes informels. La majorité des jeunes, licenciés et prêts à le (re)faire, s'approprie les modèles normatifs de ces deux modalités de pratique. Ces capacités adaptatives sont étonnantes, au creuset de la rigueur et de l'académisme fédéraux, d'une part, et des règles, des rites et des codes précis des pratiques urbaines auto-organisées, d'autre part. Ainsi, comme pour quelques autres jeunes des cités passionnés de football, le club a permis à Zidane et à Fernandez de s'accrocher à un projet, voire de bénéficier d'un capital social susceptible d'être reconverti en emploi. Ainsi en est-il d'un autre footballeur professionnel, issu comme Fernandez des Minguettes, Alim Ben Mabrouk. Échappant à l'“engrenage de la délinquance", il obtient à dixsept ans un emploi de photograveur grâce au football. À dix-huit ans, il débute sa carrière professionnelle, qui le mènera durant quatorze années sur tous les terrains hexagonaux et internationaux.

Le "capital corpo-sportif" de Platini, Fernandez et Zidane se développe et se formalise au contact des entraîneurs et animateurs sportifs de petits clubs de quartier, mais aussi du père (c'est le cas de Platini et de Zidane), qui pratique aussi le football. C'est au petit club local de l'Association sportive jovicienne que Michel Platini signe sa première licence en pupille, le 10 septembre 1966. Il ne passera pas par un des centres de formation, alors tout juste naissants en France. Fernandez commence le football de club à l'AS Minguettes, où il signe une licence portant la mention "étranger". Cette référence à son altérité, le jeune Fernandez la subit sur les terrains de football, où il est fréquemment traité d'"espinguoin", quolibet auquel il réagit souvent violemment, déclenchant des bagarres ${ }^{(30)}$. Quant à Zidane, il commence à jouer dès dix ans à l'AS Foresta (son club de quartier), puis prend sa première licence à l'US Saint-Henri. Il rejoint ensuite les Sports olympiques de Septèmes-les-Vallons puis accède au centre de formation du club de Cannes (sous la direction de Guy Lacombe). L'entrée en formation constitue à la fois une promotion sportive élective, 
marquée par un rapprochement avec l'espace strictement professionnel du football, et une rupture, plus ou moins nette, avec le cadre de la pratique et la façon de jouer antérieurs. En tant que "rite d'institution", le recrutement au centre de formation des apprentis footballeurs participe à la construction de la vocation, c'est-à-dire la croyance dans le fait "d'être fait pour ça"

$\mathrm{Au}$ contact de leurs pairs, la vocation et le capital footballistique des trois jeunes footballeurs se consolideront tout au long de leur parcours professionnel dans les clubs successifs qu'ils fréquenteront, français d'abord, européens ensuite. Naviguant entre plusieurs marchés footballistiques, ils intériorisent leurs différentes règles et apprennent à ajuster la façon de jouer. La formation ne se résume donc pas à une simple amélioration de compétences techniques, mais elle requiert aussi une transformation de la façon de jouer, nécessitant des dispositions adéquates, comme l'autodiscipline. Toutefois, au-delà d'une uniformisation, cette socialisation sportive est toujours marquée par la socialisation passée. 


\section{Capital familial et environnement social : l’exemple de Zidane}

Interviewé par Le Nouvel Observateur en décembre 2008, Zidane avoue : "Ce que je suis, je le dois à mon père et à ma mère. Je leur dois tout, par ce qu'ils m'ont appris à travailler et à être respectueux envers les autres [...]". Et d'ajouter : "Mes frères et scur, ils ont tous une situation et n'ont besoin de personne."

Issu d'une famille de cinq enfants, Zinédine raconte : "Mon père et ma mère nous ont sauvés. Ce n'est pas facile quand tu es dans un quartier, que tu subis beaucoup d'influences négatives. Sans ma famille, je ne suis rien. Sans l'éducation, sans la vision de la vie que mont donnée mes parents, jaurais pété un boulon. [...] Mon père bossait dur. Il n'avait qu'une seule après-midi pour se reposer. Au lieu de prendre du temps pour lui, il nous sortait de la cité, nous emmenait à la plage ou prendre l'air. Il était toujours derrière nous, ne nous laissait jamais trâ̂ner dans le quartier $^{(32)}$." Sauf sur la place Tartane,

\section{Bizarrement, il est peu question de l'école publique dans le parcours de Zidane. Comme si, par avance, l'école était disqualifiée en matière sportive.} entourée des immeubles de la Castellane, où Yazid jouait au football avec ses frères et copains du quartier après l'école et pendant les vacances. Avec les autres jeux de pied d'immeuble, le football participe à l'animation de la cité et occupe une grande partie du temps libre de ces jeunes garçons. Représentant 51,7\% de la population totale de la cité, les moins de vingt-cing ans sont majoritaires à la Castellane. Mais le quartier connaît aussi un important échec scolaire qui fait entrer très tôt les adolescents et les jeunes adultes sur le marché du travail. L'échec scolaire est plus imputable à l'origine ouvrière de la population du quartier ${ }^{(33)}$, majoritaire chez les parents immigrés, qu'à une culture d'origine étrangère. Les 15-25 ans sont les plus durement touchés par le chômage par rapport à la moyenne marseillaise (60,3 \% des actifs de la Castellane contre un taux de 12,9\% pour Marseille).

Malgré ce contexte, l'enfance de Zidane semble plutôt heureuse, dans un univers bien encadré par les siens et loin de la terre algérienne de ses ancêtres ; enfant, il ne s'y rendra qu'une fois, en 1985, à l'occasion d'un voyage familial. Il fréquente pourtant de nombreux autres enfants dont les parents sont originaires d'Algérie. Au collège de la Castellane, les Algériens représentent 44,52 \% de la population étrangère et 32,27 \% du total des élèves du quartier. L'effectif des élèves de la Castellane révèle la présence à la cité d'au moins 23 origines différentes ${ }^{(34)}$. 
C'est dans ce contexte multiculturel vécu que Zidane parle souvent de ses parents algériens d'origine modeste et de ses amis du quartier. Selon lui, "pour faire sa place, un étranger doit se battre deux fois plus ${ }^{(35)}$ ". Avant d'ajouter : "Mes trois mots clés : travail, sérieux, respect. Avec ça, tu vas loin, tu vas très loin ${ }^{(36)}$." La famille, le travail et le sens de l'effort personnel, la discipline et l'obéissance, la modestie, la fidélité et la solidarité : les valeurs somme toute fort traditionnelles véhiculées par Zidane semblent tout droit sorties d'un manuel d'instruction civique ${ }^{(37)}$. Bizarrement, il est peu question de l'école publique dans le parcours de Zidane. Comme si, par avance, l'école était disqualifiée en matière sportive. Même si l'on reconnaît à la "culture sportive" les vertus d'une "véritable école de la citoyenneté", sa place comme matière à part entière reste sujette à caution. Pour les "immigrés", le club est en effet souvent considéré comme une école.

\section{Conclusion}

Les exemples de Michel Platini, Luis Fernandez et Zinédine Zidane nous montrent qu'à la différence de la culture, de l'économie ou de la politique, le sport fonctionne comme un ascenseur social pour des jeunes adultes issus de l'immigration. Comme le soulignent Beaud et Noiriel, le football n'échappe pas à la logique du système français d'intégration $^{(38)}$. Si de nombreuses équipes de quartiers ont été fondées spontanément à partir de l'origine nationale (les "squadra azzura" en Lorraine, les équipes de "polacks" dans le Nord ou, plus récemment, les clubs "algériens", "portugais" ou "turcs (39)"), très vite les meilleurs éléments sont aspirés par des clubs fondés sur d'autres principes, où les origines se mélangent. Dans ces conditions, le club de football peut être considéré comme un "creuset d'intégration". Si l'on s'en tient à l'étude des vedettes du sport issues de l'immigration, on pourrait conclure à l'efficacité du rôle assimilateur du sport. Mais cette réussite (largement médiatisée) ne masque-t-elle pas une autre réalité : les discriminations vécues sur les terrains par de jeunes footballeurs ou encore les sévères désillusions de nombre d'aspirants footballeurs issus de l'immigration? La réussite des quelques footballeurs sélectionnés dans l'équipe de France de football, si elle contribue au mythe du "salut social" par le sport, fait aussi écran à la réalité de l'impasse dans laquelle se trouvent nombre de jeunes issus de l'immigration en difficulté d'insertion socioprofessionnelle. La conviction que le football serait un espace "naturel" d'intégration des immigrés ne constitue-t-elle pas finalement l'un des inconscients culturels qui fonde le sens commun? 
Bibliographie

- ABDALLAH M. H., "Leffet Zidane ou le rêve éveillé de l'intégration par le sport", Hommes et migrations $\mathrm{n}^{\circ} 1226,2000$.

- BEAUD S., NOIRIEL G., "L'immigration dans le football", Vingtième siècle n² 26, 1990, pp. 83-96.

- BOURDIEU P., Raisons pratiques. Sur la théorie de l'action, Paris, Éditions du Seuil, 1994.

- DURET P., Anthropologie de la fraternité dans les cités, Paris, PUF, 1996.

- FAURE J.-M. et SUAUD C., Le Football professionnel à la française, Paris, PUF, 1999.

- GALLOIS J.-S., PIROT P., "Football et engagement associatif des immigrés italiens et polonais en Lorraine", Migrance $\mathrm{n}^{\circ} 22,2003$.

- GASPARINI W., VIEILLE-MARCHISET G., Le Sport dans les quartiers. Pratiques sociales et politiques publiques, Paris, PUF, 2008.

- GASPARINI W., "L'intégration par le sport ?", Sociétés contemporaines n 69, Presses de Sciences Po, mars 2008.

- GASPARINI W., WEISS P., "La construction du regroupement sportif 'communautaire'. L'exemple des clubs de football turcs en France et en Allemagne", Sociétés contemporaines n 69, mars 2008, pp. 73-99.

- GASTAUT Y., "Sport et immigration : parcours individuels, histoires collectives", Migrance n² 22, 2003.

- GASTAUT Y., Le Métissage par le foot. Lintégration, mais jusqu’ò ?, Paris, Autrement, 2008.

- MAUGER G., "Précarisation et nouvelles formes d'encadrement des classes populaires", Actes de la recherche en sciences sociales $\mathrm{n}^{\circ} 136-137$, mars 2001, pp. 3-4

- MOURLANE S., "De Tarifa à Paris via les Minguettes, un parcours migratoire : Luis Fernandez", Migrance $\mathrm{n}^{\circ} 22,2003$, pp. 80-88.

- NOIRIEL G., Le Creuset français. Histoire de l'immigration, XIX ${ }^{e}-X X^{e}$ siècles. Paris, Éditions du Seuil, $1988 .$.

- SAYAD A., La Double absence. Des illusions de l'émigré aux souffrances de l'immigré, Paris, Éditions du Seuil, 1999.

- SCHWARTZ O., Le Monde privé des ouvriers. Hommes et femmes du Nord, Paris, PUF, 1990.

- TRAVERT M., L'Envers du stade. Le football, la cité et l'école, Paris, L'Harmattan, 2003

- WAHL A., LANFRANCHI P., Les Footballeurs professionnels des années trente à nos jours, Paris, Hachette, 1995.

- WILLIS P., "L'école des ouvriers", Actes de la recherche en sciences sociales n 24, 1978, pp. 50-61.

\section{Notes}

1. Né en 1955, Michel Platini est un footballeur français régulièrement cité parmi les plus grands joueurs de football de tous les temps. Après avoir été sélectionné plus de 72 fois en équipe de France $\mathrm{A}$ (capitaine à 50 reprises), il est actuellement président de l'UEFA.

2. Né en 1959 à Tarifa (Espagne), Luis Fernandez a joué en première division au Paris Saint-Germain (1978-1986), au Matra Racing Club de Paris (1986-1989) et à Cannes (1989-1992); il a été international à 60 reprises de 1982 à 1992.

3. Né en 1972 à Marseille, Zinédine Zidane fut classé à trois reprises meilleur joueur mondial de l'année par la FIFA, en 1998, 2000 et 2003, et "ballon d'or" en 1998.

4. Michel Platini, extrait d'entretien, L'Humanité, 9 décembre 2005, p. 20.

5. Voir à ce sujet William Gasparini, "Lintégration par le sport. Genèse politique d'une croyance collective", Sociétés contemporaines $\mathrm{n}^{\circ}$ 69, Presses de Sciences Po, mars 2008, pp. 7-23.

6. Le discours sur le football permet d'entretenir le culte de l'équipe, du chef et du patriotisme d'entreprise comme relais du patriotisme national ; voir Patrick Fridenson, "Les ouvriers de l'automobile et le sport", Actes de la recherche en sciences sociales, septembre 1989. 
7. Jean-Sébastien Gallois., Pierre Pirot, "Football et engagement associatif des immigrés italiens et polonais en Lorraine", Migrance n 22, 2003, p. 21.

8. Emile Durkheim, L'Éducation morale, Paris, PUF, (cours de 1902-1903), 1963.

9. Voir Didier Braun, "Football et immigration", L'Équipe, 28-31 janvier 1986.

10. Alfred Wahl, Pierre Lanfranchi, Les Footballeurs professionnels des années trente à nos jours, Paris, Hachette, 1995.

11. Luis Fernandez, Le Ballon rouge, Paris, Carrère-Michel Lafon, 1986, p. 97.

12. Stéphane Mourlane, "De Tarifa à Paris via les Minguettes, un parcours migratoire : Luis Fernandez", Migrance $\mathrm{n}^{\circ} 22,2003$, pp. 80-88.

13. Voir Yvan Gastaut, Le Métissage par le foot. Lintégration, mais jusqu'où ?, Paris, Autrement, 2008, pp. 40-41.

14. Extrait de l'interview d'un immigré kabyle, Abdelmalek Sayad, La Double absence. Des illusions de l'émigré aux souffrances de l'immigré, Paris, Seuil, 1999.

15. Benjamin Stora, Et ils venaient d'Algérie, histoire de l'immigration algérienne, Paris, Fayard, 1992.

16. William Gasparini, "L'intégration par le sport. Genèse politique d'une croyance collective", op. cit.

17. On date habituellement la naissance de la Politique de la ville en 1977 avec les dispositifs Habitat et vie sociale (HVS). Elle s'élargit dans la décennie suivante, avec la création de la Commission nationale de développement social des quartiers (CNDSQ) en 1982, la mise en place des conventions Développement social des quartiers (DSQ) à partir de 1984, puis avec la création de la délégation interministérielle à la ville (DIV) en 1988 et du ministère de la Ville en 1991, qui pilotent des dispositifs nouveaux et plus nombreux : les contrats de ville.

18. Voir à ce sujet Gérard Mauger, "Précarisation et nouvelles formes d'encadrement des classes populaires", Actes de la recherche en sciences sociales $\mathrm{n}^{\circ}$ 136-137, mars 2001, pp. 3-4.

19. Interview de Sami Naïr, Le Nouvel Observateur, 24 décembre 1998.

20. Pierre Bourdieu, Raisons pratiques. Sur la théorie de l'action, Paris, Editions du Seuil, 1994 ; Bourdieu Pierre "Comment peut-on être sportif ?", in Questions de sociologie, Paris, Éd de Minuit, 1984, pp. 173-195.

21. Maxime Travert, L'Envers du stade. Le football, la cité et l'école, Paris, L'Harmattan, 2003.

22. Thomas Sauvadet, Le Capital guerrier. Concurrence et solidarité entre jeunes de cité, Paris, Amand Colin, 2006.

23. Pascal Duret, Anthropologie de la fraternité dans les cités, Paris, PUF, 1996.

24. Cité dans L'Équipe, 28 janvier 1986.

25. Paul Willis, "L'école des ouvriers", in Actes de la recherche en sciences sociales, $n^{\circ} 24,1978$, pp. 50-61.

26. Olivier Schwartz, Le monde privé des ouvriers. Hommes et femmes du Nord, Paris, PUF, 1990, p. 478.

27. Voir Pascal Duret, Sociologie du sport, Paris, PUF, coll. "Que sais-je ?", 2008, p. 69.

28. François Dubet, La Galère. Jeunes en survie, Paris, Fayard, 1987.

29. Christian Pociello, "La force, l'énergie, la grâce et les réflexes. Le jeu complexe des dispositions culturelles et sportives", in Pociello (dir.), Sports et société : une approche socioculturelle des pratiques, Paris, Vigot,1981, pp. 171-237.

30. Dans sa biographie, Luis Fernandez raconte qu'une bagarre ainsi déclenchée sur le terrain lui vaut une suspension de six mois, (Le Ballon rouge, op. cit., p. 53).

31. Voir Jean-Michel Faure et Charles Suaud, Le Football professionnel à la française, Paris, PUF, 1999.

32. Extrait d'entretien, L'Équipe Magazine, 2 avril 2005, p. 20.

33. La surreprésentation ouvrière s'explique par le recrutement jusqu'à la fin des années quatre-vingt dans les usines alentour ou sur le port de Marseille.

34. Nadine Roudil, Normes et déviances dans l'espace urbain marseillais. Étude du mode de désignation des déviants à la cité de la Castellane, Thèse de doctorat en sociologie, université de Marseille, 2003.

35. Dan Franck, Zidane. Le roman d'une victoire, Robert Laffont-Plon, Paris, 1999.

36. Entretien avec L'Équipe Magazine, le 2 avril 2005, p. 16.

37. Mogniss H. Abdallah, "L'effet Zidane ou le rêve éveillé de l'intégration par le sport", Hommes et migrations $\mathrm{n}^{\circ} 1226,2000$, p. 9 .

38. Stéphane Beaud, Gérard Noiriel, "L'immigration dans le football", Vingtième siècle, n² 26, 1990, pp. 83-96.

39. Voir William Gasparini, Pierre Weiss, "La construction du regroupement sportif 'communautaire'. L'exemple des clubs de football Turcs en France et en Allemagne", Sociétés contemporaines n 69, mars 2008, pp. 73-99. 